\title{
Genetic Diversity and Transmitted Drug Resistance of HIV-1 Subtypes in Blood Donors Candidates in Northern Mozambique
}

\author{
Vubil $\mathbf{A}^{\mathbf{1}^{*}}$, Jani $\mathbf{V l}^{1}$, Mabunda $\mathbf{N}^{1}$, Ismael $\mathbf{N}^{1}$, Ramalho $\mathrm{D}^{1}$, Morgado $\mathbf{M G}^{2}$ and Couto-Fernandez $\mathrm{JC}^{2}$ \\ ${ }^{1}$ Instituto Nacional de Saúde, Maputo, Mozambique
}

2Laboratório de AIDS and Imunologia Molecular, Fundação Oswaldo Cruz, Rio de Janeiro, Brazil

\begin{abstract}
In order to evaluate the genetic diversity of HIV-1 subtypes and transmitted drug resistance (TDR) mutations in northern Mozambique, we analyzed 120 plasma specimens obtained from drug naive blood donors candidates, who tested positive during routine HIV screening in three blood banks in that region. The genotyping for HIV-1 resistance was performed using Trugene Genotyping System ${ }^{\mathrm{TM}}$. HIV-1 genetic subtypes were defined based on entire PR and partial RT gene regions, using REGA HIV-1 Subtyping Tool version 3.0 algorithm and confirmed by phylogenetic inference, using $\mathrm{NJ}$ algorithm. The majority of genotyped samples were classified as HIV-1 subtype C (80.0\%), followed by subtype $A 1(10.5 \%)$, subtype $D(3.2 \%)$ and subtype $G(2.1 \%)$. The inter-subtype recombinant forms $(A 1 / C, A 1 / D$ and $C / D)$ were identified in four participants (4.2\%). TDR mutations associated with nucleoside and nonnucleoside reverse transcriptase inhibitors (K219E, G190A, K101E and K103N) were observed in five (5.3\%) subjects.

Although a large proportion of HIV-1 subtype $\mathrm{C}$ was observed, non-C and recombinant forms together correspond to $20 \%$ in this study, which is different from what was described in central and southern regions, where subtype $C$ was almost $100 \%$. However, the profile mutations from this study correlate with the ARVs used both for first line schemes and PMTCT in Mozambique. This data reinforces the necessity of continuous surveillance of HIV-1 diversity, TDR and routes of spread, through inner cities of Mozambique to understand better the dynamics of the HIV-1 epidemic and support national public health policies in the country.
\end{abstract}

Keywords: Mozambique; HIV-1 genetic diversity; Resistance mutation; Blood donors

\section{Introduction}

Globally, according to the estimate from the Joint United Nation Program on HIV/AIDS (UNAIDS), at the end of 2014; about 35.3 million people were living with HIV/AIDS. Up to date, sub-Saharan African countries still remain the most affected with approximately 25 million people infected with HIV [1]. Phylogenetic analysis has revealed an extensive genetic diversity of HIV in Africa, composed of types 1 and 2, groups $\mathrm{M}, \mathrm{N}, \mathrm{O}$ and $\mathrm{P}$, subtypes $\mathrm{A}-\mathrm{K}$, circulating recombinant forms (CRFs) and many unique recombinant forms (URFs) [2-5]. Mozambique, a sub-Saharan country faces serious HIV epidemics, with an overall prevalence of $11.5 \%$ (1.6 million people) [1] within the adult population, with around one to four new infections/100 persons occurring each year [6,7]. The HIV-1 epidemic in Mozambique is mainly driven by the subtype $C$ infections [8-12]. Others non- $C$ subtypes specially subtype $\mathrm{A} 1$ and $\mathrm{D}$ have also been identified in limited number of cases mainly in the northern region [13].

In Mozambique the Antiretroviral (ART) national program was introduced in 2003 and was initially provided in two main urban cities, Maputo and Beira. Thus, a rapid scale up of people receiving highly active antiretroviral treatment (HAART) within the country was observed with a coverage that increased from $30 \%$ in 2003 to $60 \%$ in 2013.

However, due to the rapid scale up of ART, the emergence of transmitted drug resistance (TDR) is an important public health problem to be analyzed in different settings.

Thereby, the majority of TDR surveys performed in Mozambique for the past years were conducted in the central and southern regions with Maputo and Beira reporting a TDR mutation of $<5 \%$ and $5-15 \%$, respectively in 2009 [9]. In addition, there are no up-to-date data about genetic diversity and TDR available from the northern region of Mozambique. Therefore, the aim of this study was to describe the genetic diversity and the occurrence of TDR among blood donor candidates from the northern region of Mozambique.

\section{Materials and Methods}

\section{Ethical considerations}

This study was approved by the National Committee for Bioethics in Health (CNBS), Mozambique. Reference number 189/CNBS, May, $26^{\text {th }}, 2009$. Written informed consent was obtained from each participant prior to conducting any study procedure. The study was conducted according to good clinical and laboratorial practices.

\section{Study population and HIV screening}

The study was conducted in three out of four blood banks (Hospital Central de Nampula, Hospital Geral de Nacala-Porto and Hospital Provincial de Pemba) in the northern region, between November 2009 and June 2010. The blood bank of Lichinga in Niassa Province was excluded for the study because during the study period the ELISA plate reader used for HIV screening in the laboratory was broken. Initially, all enrolled participants provided a total of $500 \mathrm{ml}$ of whole

*Corresponding author: Adolfo Vubil, Eduardo Mondlane Avenue, Ministry of Health Building, Maputo-Mozambique, Tel: +258828285630; Fax: +25821309317; E-mail: avubil@gmail.com

Received September 19, 2016; Accepted October 03, 2016; Published October 10, 2016

Citation: Vubil A, Jani VI, Mabunda N, Ismael N, Ramalho D, et al. (2016) Genetic Diversity and Transmitted Drug Resistance of HIV-1 Subtypes in Blood Donors Candidates in Northern Mozambique. J AIDS Clin Res 7: 623. doi: 10.4172/21556113.1000623

Copyright: (C) 2016 Vubil A, et al. This is an open-access article distributed unde the terms of the Creative Commons Attribution License, which permits unrestricted use, distribution, and reproduction in any medium, provided the original author and source are credited. 
blood in a blood bag. The specimens were screened for HIV according to the national algorithm, using Genscreen ${ }^{\mathrm{Tm}} \mathrm{HIV}-1 / 2$ version 2, (Bio-Rad, France) and Determine HIV-1/2 (Alere, Germany). For the HIV positive samples, $50 \mu \mathrm{l}$ of whole blood was used to prepare Dried Blood Spots (DBS) and $6.0 \mathrm{ml}$ of plasma were prepared for each participant. All plasma and DBS specimens were sent to the reference laboratory of the Instituto Nacional de Saúde in Maputo, Mozambique where a confirmatory test was performed in plasma specimens, using Genscreen $^{\text {Tw }}$ HIV-1/2 version 2, (Bio-Rad, France;) Murex HIV Ag/Ab combination, (Abbott, UK) and Amplicor HIV-1 DNA test, version 1.5 (Roche Diagnostics, Germany) was performed in DBS specimens. For the specimens that were confirmed positive at the reference laboratory, viral load was measured in plasma specimen using COBAS Ampliprep/ TaqMan'96 version 2.0, (Roche Diagnostics, Germany). The remaining sample portion was stored and later was used to prepare Proficiency Testing Panel at National Institute of Health Reference Laboratory. The permission for it was asked from the participants during the enrolment for the study.

\section{RNA extraction, RT-PCR and sequencing for genotyping}

HIV RNA was extracted from 140 ul of plasma, using QIAamp RNA Mini Kit, according to manufacturer's instructions (QIAGEN, USA). The RT-PCR, one-step PCR and sequencing was performed using Trugene HIV-1 Genotyping System, (Siemens Diagnostics, US), according to manufacturer's instructions and the sequences were analyzed using OpenGene DNA software.

\section{HIV-1 subtyping, phylogenetic and resistance mutation analysis}

For subtype analysis the sequences were submitted to the REGA HIV-1 Subtyping Tool version $3.0[14,15]$ and confirmed with phylogenetic analysis, where the nucleotide pol gene sequences from this study were aligned together with representative reference sequences (group M subtypes A, B, C, D and G) retrieved from Los Alamos HIV Sequence Database [16], using ClustalW algorithm, available through MEGA version 5.0 software [17]. Tamura-Nei was the best fitting substitution model [18] with 100 replicates bootstrap and group $\mathrm{O}$ was used to root the phylogenetic tree. Neighbor-joining (NJ) algorithm [15] was used for phylogenetic inferences of all pure subtypes from this study.

Furthermore, to investigate routes of transmission of subtype C, based on virus similarity, the Subtype C Maximum Likelihood (ML) tree was performed using PhyML 3.0 algorithm [19], GTR was the best fitting substitution model [20], and SPR was applied to search the tree space. Recombination analysis was performed by bootscanning, using SimPlot.

Transmitted Drug Resistance mutations were analyzed using the Calibrated Population Resistance Tool (CPR) [21].

\section{Results}

\section{Study population}

From November 2009 through June 2010, a total of 200 blood donors were included in this study. Of these, 120 (60\%) were serologically reactive for HIV-1 and confirmed by Amplicor HIV-1 DNA test version 1.5.

The median age of participants was 29 years old, and, in terms of gender distribution, all were males donating blood for the first time. None of the participants knew their HIV status. The main reason for donation was to replace the stock at the blood bank due to a transfusion that had to be done for a relative. Plasma viral load was high in most infected subjects with a mean of $5.0 \log 10$. Ninety two (76.7\%) participants were from Nampula city, seventeen (14.2\%) from Pemba, and eleven $(9.2 \%)$ from Nacala Porto.

\section{HIV-1 genetic diversity}

Ninety five (79.2\%) out of 120 samples were successfully genotyped for HIV-1 resistance, generating good quality sequences from HIV pol gene (entire Protease and partial RT). Among the 95 sequences submitted for phylogenetic analysis, $91(95,8 \%)$ were classified as HIV1 group M pure subtypes (C, A1, D and G) and 4 (4.2\%) were classified as recombinant forms $(\mathrm{A} 1 / \mathrm{C}, \mathrm{A} 1 / \mathrm{D}$ and $\mathrm{C} / \mathrm{D})$. Boot scanning analysis of concatenated genes, using Simplot confirmed the recombination events (Figure 1).

According to the REGA HIV-1 Subtyping Tool analysis, confirmed by phylogenetic analysis, $76(80.0 \%)$ sequences clustered with subtype C, ten $(10.5 \%)$ with subtype A1, three (3.2\%) with subtype D and
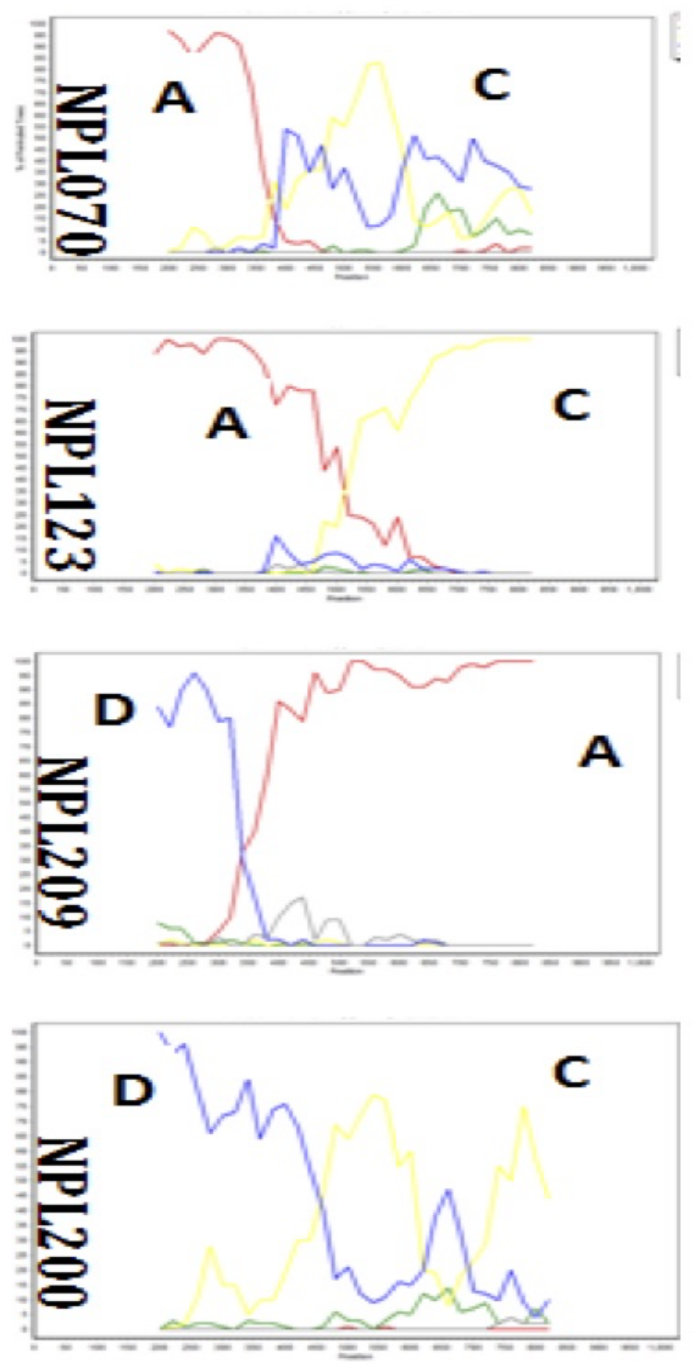

Figure 1: Four isolates from the study: NPL200, NPL 209, NPL 123 and NPL070, did not cluster in HIV pure subtypes. They were aligned with HIV-1 subtype A, B, C, D and G. Simplot analysis were performed for each isolate and are illustrated above. Red line indicates subtype A strains, yellow subtype $\mathrm{C}$ and blue subtype $\mathrm{D}$. 
two (2.1\%) sequences with subtype $\mathrm{G}$ (Table 1$)$. In addition, for the Maximum Likelihood analysis of subtype $\mathrm{C}$ a large dataset of 1786 subtype $\mathrm{C}$ reference sequences from south and east African countries, available in full or near full length genome in the Los Alamos HIV Database, were aligned together with 75 pure subtype C sequences from our study. In this analysis was observed that the subtype $\mathrm{C}$ sequences from Mozambique, including references, were segregate in small phylogenetic sub clusters, showing an almost uniform distribution along the phylogenetic tree (Figure 2). The largest cluster involving Mozambican strains were observed mainly with South African sequences. In contrast, subtype $\mathrm{C}$ strains recovered in eastern African countries (Uganda, Tanzania, Kenya and Burundi) seems to be relatively more diverse and clustered in a separate group.

\section{Drug resistance mutations and other polymorphisms}

Among the 95 blood donors analyzed, 19 (20.0\%) have shown at least one major or minor mutation in the RT or PR region. The CPR analysis identified four mutations in five subjects (5.3\%) as surveillance drug resistance mutation (SDRM) [21], occurring alone or combined each other. Four out of five subjects with SDRM were carrying NNRTIs mutations (K103N, n=1; K101E, n=2; G190A, n=2) and one NRTI (K219E) was found in one subject (Table 2). The mutations G190A and $\mathrm{K} 103 \mathrm{~N}$ are known to confer high-level resistance to Efavirenz (EFV) and Nevirapine (NVP), while K101E causes intermediatelevel resistance to NVP and low-level to EFV. On the other hand, the Timidin analogue mutation (K219E) reported in one subject is known to be associated with decreased susceptibility to Zidovudine (AZT) and

\begin{tabular}{|c|c|c|c|c|}
\hline Subtypes & Nampula & Nacala porto & Pemba & Total \\
\hline C & $67(83.8 \%)$ & $2(66.7)$ & $6(50.0 \%)$ & 75 \\
\hline A1 & $6(7.5 \%)$ & - & $4(33.3 \%)$ & 10 \\
\hline D & $2(2.5 \%)$ & $1(33.3)$ & - & 3 \\
\hline G & - & - & $2(16.7 \%)$ & 2 \\
\hline URFs & $5(6.3 \%)$ & - & - & 5 \\
\hline Total & 80 & 3 & 12 & 95 \\
\hline
\end{tabular}

Table 1: HIV-1 Subtypes distribution per cities.

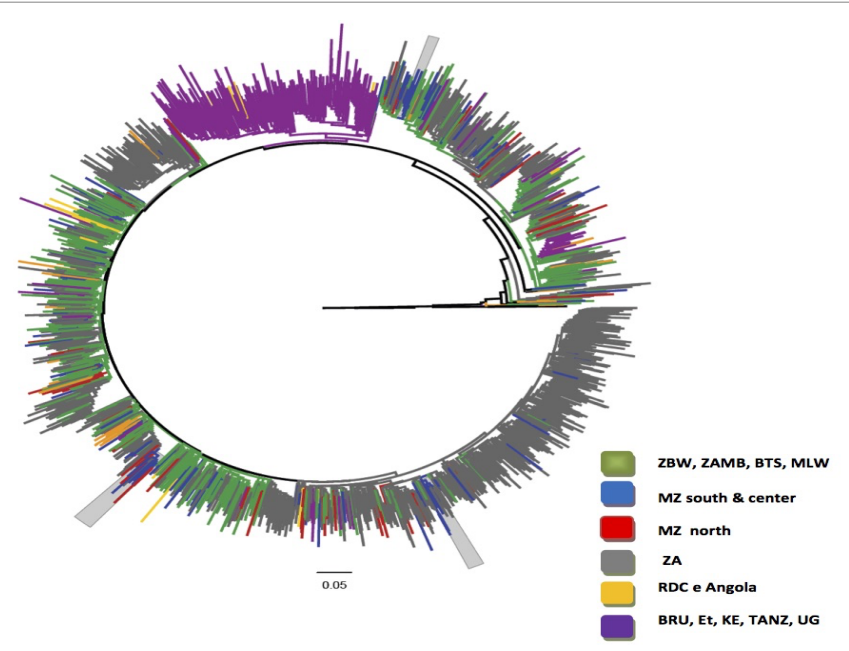

Figure 2: Phylogenetic analysis of HIV-1 subtype $C$ sequences from blood donors retrived in Nampula, Nacala Porto and Pemba, northern Mozambique. 75 pure subtype $\mathrm{C}$ sequences of HIV-1, entire protease and partial RT genes (codon 40-247) from our study, were aligned with 1711 pure subtype $C$ from southern, central and east Africa. Colors were used to identify sequences from same region or country. Group $\mathrm{O}$ was used as out group. The Maximum Likelihood tree was constructed using PhyML 3,0. GTR substitution model was applied, with 100 replicates bootstrap. possibly to Stavudine $[22,23]$. Beside the SDRM, were also observed in the RT region, the minor mutations $(\mathrm{K} 101 \mathrm{H}$ and V106I in one subject each and E138A in nine subjects). Both $\mathrm{K} 101 \mathrm{H}$ and E138A are common accessory polymorphic mutations associated with decreased susceptibility to two second generation NNRTIs (Rilpivirine and Etravirine) [22]. Additionally, $\mathrm{K} 101 \mathrm{H}$ is also associated with decreased response to NVP and EFV [22,24]. No major mutations associated with resistance to protease inhibitors (PIs) was identified in our study. However, five minor PI mutations were observed (L10I, $\mathrm{n}=1$; K20I, $\mathrm{n}=2$; V11I, $\mathrm{n}=1$; T74S, $\mathrm{n}=6$; L10F, $\mathrm{n}=1$ ). The combination of T74S with E138A observed in two subjects could cause low-level resistance to Rilpivirine and Nelfinavir, while two participants harboring only T74S, display low level resistance to Nelfinavir [22]. In one participant, the mutation K20I was associated with V11I, causing low-level resistance to Nelfinavir and potential low-level was observed when combined with L10I (Table 2).

Although not considered in WHO SDRM list, several polymorphisms listed in the International Antiviral Society (IAS) (2014) panel were observed in the PI region, of which M36I (83.2\%); H69K (94.7\%); L89M (83.2\%) and I93L (74.7\%) (Figure 3).

\section{Discussion}

\section{HIV-1 genetic diversity}

In this study we found that HIV subtype $C$ is the most frequent in northern Mozambique. However, other subtypes (A1, D, G) and recombinant forms were also detected. The finding of subtype $\mathrm{C}$ in three different cities from northern Mozambique reinforce the hypothesis that this subtype has been circulating as a major lineage, not only in the northern of Mozambique but also in the south and center of the country $[8,9]$. In fact, there is strong evidence of subtype C epidemic spread throughout the country $[8-10,12,25]$, as well as in other southern African countries [26,27].

Additionally, in a separated phylogenetic analysis of pure subtype C, strains from Mozambique clustered mainly with South African strains, which suggest an epidemiological linkage between these countries. These findings are in line with Msimanga et al., who discuss the impact of migratory movements in the spread of epidemics [26]. Our result is also consistent with Baltazar et al. who reported $22.3 \%$ HIV prevalence in Mozambican mine workers in South Africa [28]. These findings are consistent; given the intensive migratory flux due to labor necessity mainly of Mozambican mine workers in South Africa. In addition, the intensive flux of truck drivers through the Maputo corridor, which connects South Africa to Maputo Port in the south and to the central provinces (Manica, Tete, Zambézia), may be a favorable occasion of virus spread in the region [26].

In our study, HIV-1 non-C subtypes $(\mathrm{A} 1, \mathrm{D}, \mathrm{G})$ together with recombinants, were reported in a relatively high frequency $(20 \%)$. Moreover, in Pemba, non-C subtypes from our study represented 50\% (6 of 12) of the total subjects analyzed. This finding is in line with Abreu et al. who reported high diversity of HIV in the same region in 2002 [13]. However, our results are different from previous studies, which reported almost $100 \%$ subtype $\mathrm{C}$ in central and southern Mozambique $[8,9,25]$. These findings emphasize that although the HIV-1 epidemic in Mozambique is dominated by subtype $\mathrm{C}$, non-C subtypes (A1, $\mathrm{D}, \mathrm{G})$ and inter-subtype combination are prominent features to be considered in northern region. The HIV epidemic pattern reported in northern Mozambique may somehow reflect the influence of A1 and D subtypes epidemic in east African countries (Kenya, Rwanda, Tanzania 
Page 4 of 6

\begin{tabular}{|c|c|c|c|c|c|c|c|}
\hline \multirow[b]{2}{*}{ Sample ID } & \multirow[b]{2}{*}{ Subtype } & \multicolumn{2}{|c|}{ Resistance Mutations } & \multicolumn{4}{|c|}{ Resistance Level } \\
\hline & & PR & RT & Potential Low & Low & Intermediate & High \\
\hline MZ.2010.P001 & G & L10I, K20I & None & NFV & & & \\
\hline MZ.2010.P013 & $\mathrm{A} 1$ & None & $\mathrm{K} 101 \mathrm{H}, \mathbf{G} 190 \mathrm{~A}$ & & ETR, RPV & & EFV, NVP \\
\hline MZ.2010.P004 & G & V11I, K20I & None & & NFV & & \\
\hline MZ.2010.NPL190 & $\mathrm{D}$ & None & K101E, G190A & & & ETR, RPV & EFV, NVP \\
\hline MZ.2010.NP004 & $\mathrm{C}$ & T74S & E138A & & NFV, ETR, RPV & & \\
\hline MZ.2010.NP008 & C & T74S & None & & NFV & & \\
\hline MZ.2010.P005 & C & None & K219E, E138A & ETR & RPV & & \\
\hline MZ.2010.P008 & $\mathrm{C}$ & T74S & E138A & ETR & NFV, RPV & & \\
\hline MZ.2010.P016 & $\mathrm{C}$ & None & K103N & & & & EFV, NVP \\
\hline MZ.2010.P019 & $\mathrm{C}$ & None & E138A & ETR & RPV & & \\
\hline MZ.2010.NPL048 & $\mathrm{C}$ & T74S & None & & NFV & & \\
\hline MZ.2010.NPL051 & $\mathrm{A} 1$ & & V106I & & & & \\
\hline MZ.2010.NPL071 & C & None & E138A & & ETR, RPV & & \\
\hline MZ.2010.NPL127 & C & T74S & None & & NFV & & \\
\hline MZ.2010.NPL131 & C & L10F & None & $\mathrm{FPV} / \mathrm{r}, \mathrm{IDV} / \mathrm{r}, \mathrm{NFV}$ & & & \\
\hline MZ.2010.NPL140 & $\mathrm{C}$ & None & E138A & ETR & RPV & & \\
\hline MZ.2010.NPL160 & $\mathrm{C}$ & None & E138A & ETR & RPV & & \\
\hline MZ.2010.NPL170 & C & None & E138A & ETR & RPV & & \\
\hline MZ.2010.NPL235 & $\mathrm{C}$ & T74S & None & & NFV & & \\
\hline MZ.2010.NPL240 & $\mathrm{C}$ & None & E138A, K101E & & EFV, ETR; & NVP, RPV & \\
\hline
\end{tabular}

Table 2: HIV-1 drug resistance profile mutation.

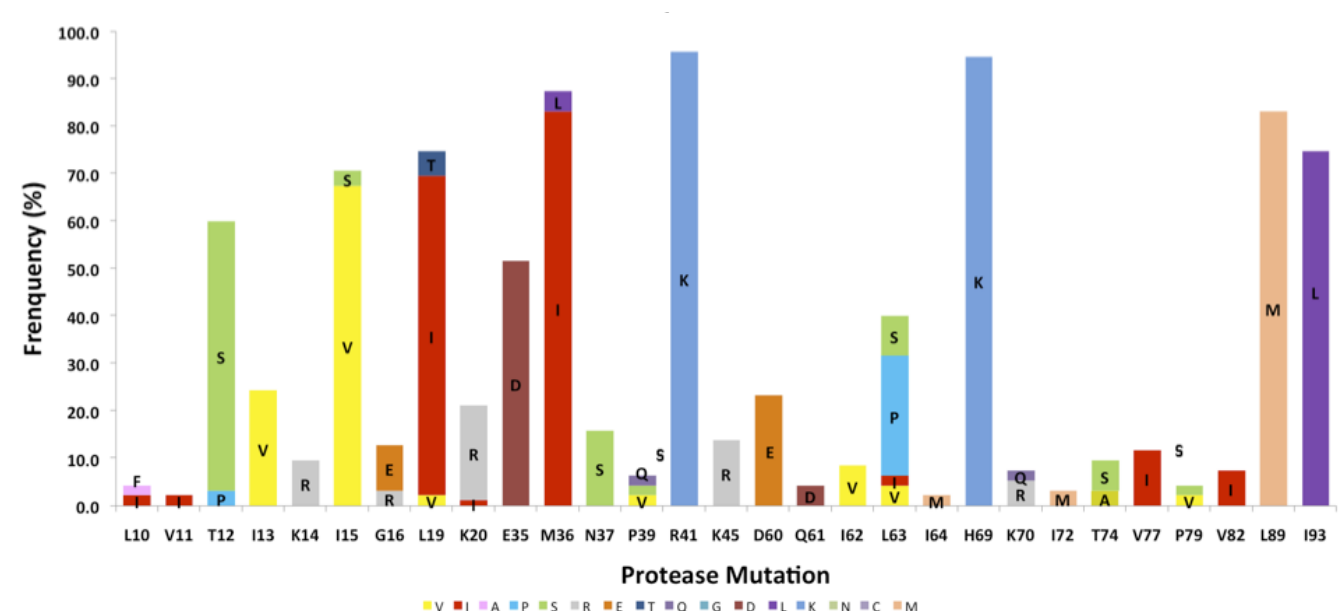

Figure 3: Frequency of polymorphism within protease gene in 95 blood donors sequences collected in Northern Mozambique.

and Uganda) [27]. In fact this finding is plausible given the historical relationship, which allows intensive flux of people between countries.

Overall, results from phylogenetic analysis of subtype C, A1, D and $G$ from this study, suggest that Mozambique has an epidemiological linkage with most southern African countries, characterized by multiple introductions and circulation of HIV-1 strains in the country. This finding was also observed by Bartolo et al. in southern Mozambique in 2009 [25]. However, advanced phylogenetic analysis and continued monitoring of the different strains is necessary to understand better the origin of the strains circulating in northern Mozambique and also the possible spread to the central and southern regions of the country. The introduction of additional subtypes may lead to the formation of novel recombinant forms and the emergence of variants with biological and epidemiological property changes. The CRF01_AE is one of the most studied and has shown to be more pathogenic than pure subtype B in Singapore. A changing of molecular epidemiology profile may have important consequences on diagnostic testing, therapeutic response to treatment and vaccine candidates design $[29,30]$.

\section{Drug resistance mutations and other polymorphisms}

In this study, we found evidence of HIVDR mutations for the first time in the northern region, and it is the first report of HIVDR mutations in blood donor candidates in Mozambique. For both $R T$ and $P R$, SDRM level was low. This finding is in line with Bila et al. who reported in naïve pregnant women in 2009 a low level $(<5.0 \%)$ of TDR in southern, while an intermediate level (5.0-15.0\%) was reported in central Mozambique [9]. On the other hand, data available, from naive pregnant women did not report any TDR in southern, central, and northern Mozambique in 2002, but high molecular diversity of HIV-1 subtypes was described in the northern region [13]. All together this data shows that TDR has been emerging in Mozambique. The trend of TDR elucidated in this study is consistent to global studies which indicate low prevalence of TDR in sub-Saharan Africa when compared with North America, Latin America and Europe [31-33], but with increasing trend from 2004 to 2010 [33,34]. The mutations K103N and K101E observed in our study were described in previous study 
Citation: Vubil A, Jani VI, Mabunda N, Ismael N, Ramalho D, et al. (2016) Genetic Diversity and Transmitted Drug Resistance of HIV-1 Subtypes in Blood Donors Candidates in Northern Mozambique. J AIDS Clin Res 7: 623. doi: 10.4172/2155-6113.1000623

in pregnant women naive to ARVs in Beira and E138A in Beira and Maputo in 2009 [9]. The occurrence of K103N and G190A will inhibit the use of Nevirapine and Efavirenz drugs, which are the backbone of ART first-line schemes in Mozambique. Thereby mutations will force inclusion of PI inhibitors in first-line schemes, which will further limit therapeutic schemes options for the second line. Additionally, the occurrence of E138A can compromise the use of NNRTIs second generation (Rilpivirine and Etravirine) in first line schemes. This scenario may compromise treatment strategies, especially in resourcepoor countries. Furthermore, the mutation T74S, reported in this study is a common polymorphism in subtype $\mathrm{C}$, which is known to be associated with restoring fitness of multi-drug resistant virus in vitro [35].

The polymorphisms M36I, H69K, L89M and I93L reported in our study were also observed in high frequency in other studies evolving non-B subtypes and are considered as polymorphic signatures for non-B subtypes virus $[9,25]$.

Our study is a strong evidence of TDR mutation circulation in northern Mozambique. However, continued surveys with nationally representative pre-treatment patients could provide more details to inform first line schemes guidelines in a national context, leading to an increase of ARV access in the country.

\section{Acknowledgement}

This study was supported by Instituto Nacional de Saúde (INS), Mozambique; Fundo Nacional de Investigação (FNI), Mozambique; IOC-FIOCRUZ, The Brazilian Network for HIV-1 Genotyping (Renageno) provided invaluable technical support. We wish to thank our participants, for accepting to participate in the study. Sequences were deposited on the GenBank database under the accession numbers KT729409 to KT729503.

\section{References}

1. UNAIDS (2013) Global Report 2013.

2. Sharp PM, Hahn BH (2011) Origins of HIV and the AIDS pandemic. Cold Spring Harb Perspect Med 1: a006841.

3. Keele BF, Van Heuverswyn F, Li Y, Bailes E, Takehisa J, et al. (2006) Chimpanzee reservoirs of pandemic and non-pandemic HIV-1. Science 313 : 523-526.

4. Bredell H, Hunt G, Casteling A, Cilliers T, Rademeyer C, et al. (2002) HIV-1 Subtype A, D, G, AG and unclassified sequences identified in South Africa. AIDS Res Hum Retroviruses 18: 681-683.

5. Alamos L (2014) HIV sequence compendium.

6. González R, Augusto OJ, Munguambe K, Pierrat C, Pedro EN, et al. (2015) HIV incidence and spatial clustering in a rural area of Southern Mozambique. PLoS One 10: e0132053.

7. Viegas EO, Tembe N, Macovela E, Gonçalves E, Augusto O, et al. (2015) Incidence of HIV and the prevalence of HIV, hepatitis B and syphilis among youths in Maputo, Mozambique: A cohort study. PLoS One 10: e0121452.

8. Bila DC, Boullosa LT, Vubil AS, Mabunda NJ, Abreu CM, et al. (2015) Trends in prevalence of HIV-1 drug resistance in a public clinic in Maputo, Mozambique. PLoS One 10: e0130580.

9. Bila DC, Young P, Merks H, Vubil AS, Mahomed M, et al. (2013) Evolution of primary HIV drug resistance in a subtype $\mathrm{C}$ dominated epidemic in Mozambique. PLoS One 8: e68213.

10. Bellocchi MC, Forbici F, Palombi L, Gori C, Coelho E, et al. (2005) Subtype analysis and mutations to antiviral drugs in HIV-1-infected patients from Mozambique before initiation of antiretroviral therapy: Results from the DREAM programme. J Med Virol 76: 452-458.

11. Parreira R, Piedade J, Domingues A, Lobao D, Santos M, et al. (2006) Genetic characterization of human immunodeficiency virus type 1 from Beira, Mozambique. Microbes Infect 8: 2442-2451.
12. Vaz P, Augusto O, Bila D, Macassa E, Vubil A, et al. (2012) Surveillance of HIV drug resistance in children receiving antiretroviral therapy: A pilot study of the World Health Organization's generic protocol in Maputo, Mozambique. Clin Infect Dis 54: S369-S374.

13. Abreu CM, Brindeiro PA, Martins AN, Arruda MB, Bule E, et al. (2008) Genotypic and phenotypic characterization of human immunodeficiency virus type 1 isolates circulating in pregnant women from Mozambique. Arch Virol 153: 2013-2017.

14. Alcantara LC, Cassol S, Libin P, Deforche K, Pybus OG, et al. (2009) A standardized framework for accurate, high-throughput genotyping of recombinant and non-recombinant viral sequences. Nucleic Acids Res 37: W634-W642.

15. de Oliveira T, Deforche K, Cassol S, Salminen M, Paraskevis D, et al. (2005) An automated genotyping system for analysis of HIV-1 and other microbial sequences. Bioinformatics 21: 3797-3800.

16. Yoon H, Leitner T (2015) PrimerDesign-M: A multiple-alignment based multiple-primer design tool for walking across variable genomes. Bioinformatics 31: $1472-1474$.

17. Tamura K, Peterson D, Peterson N, Stecher G, Nei M, et al. (2011) MEGA5: Molecular evolutionary genetics analysis using maximum likelihood, evolutionary distance and maximum parsimony methods. Mol Biol Evol 28: 2731-2739.

18. Tamura K, Dudley J, Nei M, Kumar S (2007) MEGA4: Molecular evolutionary genetics analysis (MEGA) software version 4.0. Mol Biol Evol 24: 1596-1599.

19. Guindon S, Dufayard JF, Lefort V, Anisimova M, Hordijk W, et al. (2010) New algorithms and methods to estimate maximum-likelihood phylogenies: Assessing the performance of PhyML 3.0. Syst Biol 59: 307-321.

20. Felsenstein $\mathrm{J}$ (1981) Evolutionary trees from DNA sequences: A maximum likelihood approach. J Mol Evol 17: 368-376.

21. Gifford RJ, Liu TF, Rhee SY, Kiuchi M, Hue S, et al. (2009) The calibrated population resistance tool: Standardized genotypic estimation of transmitted HIV-1 drug resistance. Bioinformatics 25: 1197-1198.

22. Liu TF, Shafer RW (2006) Web resources for HIV type 1 genotypic-resistance test interpretation. Clin Infect Dis 42: 1608-1618.

23. García-Lerma JG, MacInnes H, Bennett D, Weinstock H, Heneine W (2004) Transmitted human immunodeficiency virus type 1 carrying the $\mathrm{D} 67 \mathrm{~N}$ or K219Q/E mutation evolves rapidly to zidovudine resistance in vitro and shows a high replicative fitness in the presence of zidovudine. J Virol 78: 7545-7552.

24. Melikian GL, Rhee SY, Varghese V, Porter D, White K, et al. (2014) Nonnucleoside reverse transcriptase inhibitor (NNRTI) cross-resistance: Implications for preclinical evaluation of novel NNRTIs and clinical genotypic resistance testing. J Antimicrob Chemother 69: 12-20.

25. Bártolo I, Casanovas J, Bastos R, Rocha C, Abecasis AB, et al. (2009) HIV1 genetic diversity and transmitted drug resistance in health care settings in Maputo, Mozambique. J Acquir Immune Defic Syndr 51: 323-331.

26. Msimanga PW, Vardas E, Engelbrecht S (2015) HIV-1 diversity in an antiretroviral treatment naive cohort from Bushbuckridge, Mpumalanga Province, South Africa. Virol J 12: 24.

27. Tatem AJ, Hemelaar J, Gray RR, Salemi M (2012) Spatial accessibility and the spread of HIV-1 subtypes and recombinants. AIDS 26: 2351-2360.

28. Baltazar CS, Horth R, Inguane C, Sathane I, César F, et al. (2015) HIV prevalence and risk behaviors among Mozambicans working in South African mines. AIDS Behav 19 Suppl 1: S59-S67.

29. Lessells RJ, Katzenstein DK, de Oliveira T (2012) Are subtype differences important in HIV drug resistance? Curr Opin Virol 2: 636-643.

30. Bruzzone B, Ventura A, Bisio F, Mboungou FA, Miguel LM, et al. (2010) Impact of extensive HIV-1 variability on molecular diagnosis in the Congo basin. J Clin Virol 47: 372-375.

31. Baxter JD, Dunn D, White E, Sharma S, Geretti AM, et al. (2015) Global HIV1 transmitted drug resistance in the INSIGHT strategic timing of antiretroviral treatment (START) trial. HIV Med 16: 77-87. 
Citation: Vubil A, Jani VI, Mabunda N, Ismael N, Ramalho D, et al. (2016) Genetic Diversity and Transmitted Drug Resistance of HIV-1 Subtypes in Blood Donors Candidates in Northern Mozambique. J AIDS Clin Res 7: 623. doi: 10.4172/2155-6113.1000623

Page 6 of 6

32. Frentz D, Boucher CA, van de Vijver DA (2012) Temporal changes in the epidemiology of transmission of drug-resistant HIV-1 across the world. AIDS Rev 14: 17-27.

33. Rhee SY, Blanco JL, Jordan MR, Taylor J, Lemey P, et al. (2015) Geographic and temporal trends in the molecular epidemiology and genetic mechanisms of transmitted HIV-1 drug resistance: An individual-patient- and sequence-level meta-analysis. PLoS Med 12: e1001810.
34. Bennett DE, Camacho RJ, Otelea D, Dam O, Daniel RK, et al. (2009) Drug resistance mutations for surveillance of transmitted HIV-1 drug-resistance: 2009 update. PLoS One 4: e4724.

35. Soares EA, Santos AF, Gonzalez LM, Lalonde MS, Tebit DM, et al. (2009) Mutation T74S in HIV-1 subtype B and C proteases resensitizes them to ritonavir and indinavir and confers fitness advantage. J Antimicrob Chemother 64: 938-944 